\title{
The Sunyaev-Zel'dovich Effect of Nearby Clusters in the 3-Year WMAP Data
}

\author{
Jian $\mathbf{H u}^{1}$, Jianyin $\mathbf{N i e}^{1,2}$, and Shuangnan Zhang ${ }^{1,2,3}$ \\ ${ }^{1}$ Physics Department and Tsinghua Center for Astrophysics, Tsinghua University, \\ Beijing 100084, China. email: hujian02@mails.thu.edu.cn \\ ${ }^{2}$ Institute of High Energy Physics, Chinese Academy of Sciences, \\ P.O. Box 918-3, Beijing 100039, China \\ ${ }^{3}$ Department of Physics, University of Alabama in Huntsville, Huntsville, AL 35899, USA
}

\begin{abstract}
We explore the thermal Sunyaev-Zel'dovich (SZ) effect of a sample of 31 clusters in the three-year data of the Wilkinson Microwave Anisotropy Probe (WMAP), and detect strong SZ signal at the $\sim 10 \sigma$ level. By comparing the co-added SZ profile of the sample clusters with the prediction based on the X-ray observations of ROSAT and XMM-Newton, we find the $\mathrm{SZ}$ profiles are consistent with the isothermal $\beta$-model of intracluster medium (ICM), but the central region $\left(<0^{\circ} .2\right)$ may be contaminated by the central radio sources in the clusters. We derive a mean outer radius of the ICM distribution at $\sim 1.2 \pm 0.4 r_{\text {vir }}(68 \% \mathrm{CL})$. The existence of the ICM outer edge could be the first evidence of ICM accretion shock, which is important for understanding the formation and evolution of clusters. We also estimate the mean gas mass fraction of the ICM from the overall amplitude of the SZ signals as $f_{\text {gas }}=0.10 \pm 0.05$, which is consistent with the X-ray observation and lower than the cosmic average baryon fraction.
\end{abstract}

Keywords. cosmic microwave background, galaxy cluster

\section{Introduction}

The Sunyaev-Zel'dovich effect (SZE), caused by the scattering of cosmic microwave background photons by the hot gas in the intracluster medium (ICM), is a powerful tool to probe the astrophysical process in galaxy clusters (e.g. the formation and evolution of the clusters and intracluster medium) and determine the cosmological parameters (e.g. the Hubble constant and baryon fraction, for recent reviews, cf. Birkinshaw 1999; Carlstrom et al. 2002). Since the first-year data release of WMAP in 2003, many efforts have been made to detect SZ signals in the WMAP CMB maps. There are two main approaches. The first method is cross-correlation analysis between the CMB map with the galaxy or cluster surveys which trace the density fluctuation in the low-redshift universe, the other method is to detect SZ signals based on the templates made out of the galaxy/Cluster (Afshordi et al. 2004 and reference therein).

In this paper, we follow the method of Lieu et al. (2006) but use the three-year WMAP data to detect SZE signal in a catalogue of 31 clusters at high Galactic latitude $\left(|b|>30^{\circ}\right)$ with low column density of Galactic neutral hydrogen.

The detail of our analysis and results can be found in $\mathrm{Hu}$ et al. (2006).

\section{References}

Afshordi, N., Loh, Y.-S., \& Strauss, M. A. 2004, Phys. Rev. D 69, 083524.

Birkinshaw, M. 1999, Phys. Rep. 310, 97.

Carlstrom, J., Holder, G., \& Reese, E. 2002, ARA\&A 40, 643.

Hu, J., Nie, J.-Y, \& Zhang, S. N. 2006, ApJ Letter submitted.

Lieu, R., Mittaz, J. P. D., \& Zhang, S. N. 2006, ApJ 648, 176. 